\title{
Photometric Observations of Eccentric Accretion in Algol-type Binary Stars
}

\author{
Phillip A. Reed ${ }^{\dagger}$ \\ Department of Physical Sciences, Kutztown University of Pennsylvania, Kutztown, PA 19530, USA
}

Some Algol-type interacting binary stars exhibit strange photometric variations that can be phase-dependent and/or secular. This paper discusses the possibility of explaining these observed variations as resulting from an accretion structure eclipsing one or both of the stars. Some previous studies are reviewed and suggestions for future work are made, including the prospective of incorporating data from the Kepler Observatory.

Keywords: interacting binaries, accretion, photometry, R Arae

\section{INTRODUCTION}

Intermediate-period Algols (those with orbital periods between approximately 3 and 5 days) are not close enough to experience direct impact of accretion from one star to the other, but are perhaps too close for a stable accretion disk to form. As a result, we might expect to find variable, transient, and non-circular accretion structures in these systems.

Here we review a study of the neglected southern interacting Algol-type binary R Ara, which modeled spectral blending and photometric variations as effects due to an accretion structure present in the system. In addition to R Ara, there are several other similar systems that could benefit from further observation in order to determine if variable and eccentric accretion can explain their photometric variations.

\section{THE CASE OF R ARAE}

A good example to discuss is $\mathrm{R}$ Ara, whose orbital period is 4.4 days. After its discovery in 1892, $\mathrm{R}$ Ara was observed spectroscopically by Sahade (1952) and photometrically by groups in New Zealand (Nield et al. 1986, Forbes et al. 1988, Banks 1990, Nield 1991). Sahade reported badly blended absorption features probably resulting from mass transfer, and the photometric studies found strange variations that couldn't be explained in the light curve models.

A more recent study by Reed et al. (2010) made use of the archival International Ultraviolet Explorer (IUE) satellite data and developed a model of a variable and eccentric accretion structure that explained the spectral line blending as well as the photometric variations. Reed (2011) also obtained new observations and combined them with those in the available literature to construct the first ephemeris

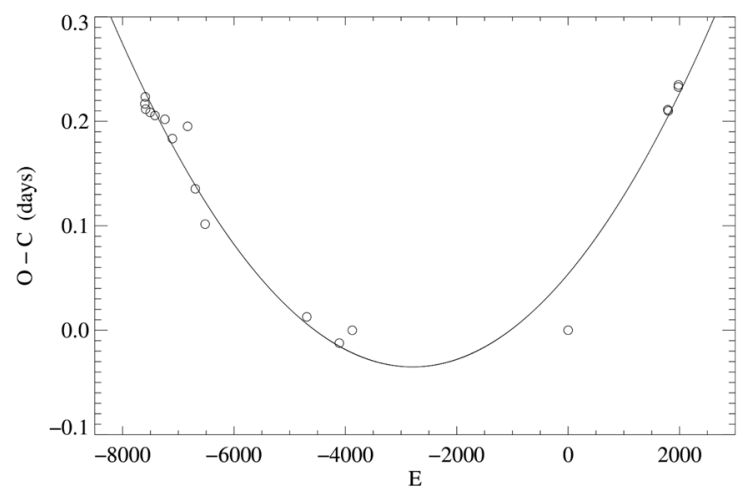

Fig. 1. The ephemeris curve for R Ara, spanning 116 years since its discovery. Reprinted from Reed (2011).

Received Mar 2, 2012 Revised Apr 13, 2012 Accepted May 1, 2012 †Corresponding Author E-mail: preed@Kutztown.edu Tel: +1-610-683-4438 Fax: +1-610-683-1352 


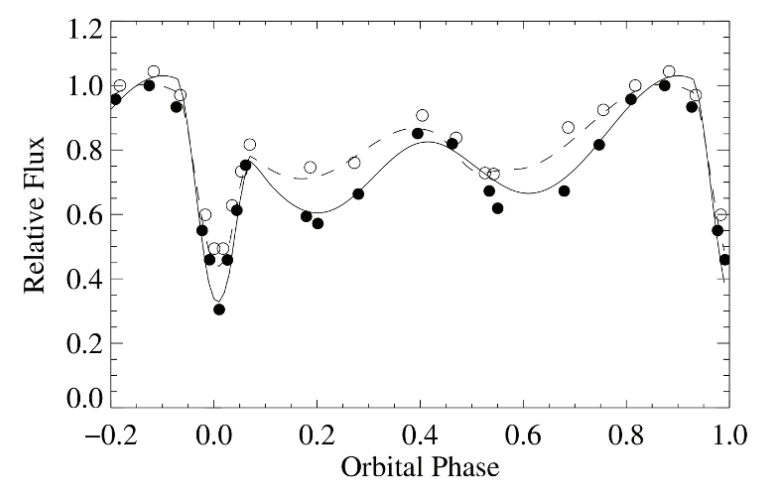

Fig. 2. The International Ultraviolet Explorer light curve for Ara using data from 1989. The solid circles are relative fluxes measured at $1,320 \AA$ and the open circles are at 2,915 $\AA$. The solid and dashed lines correspond to the model (with eccentric accretion). Reprinted from Reed et al. (2010).

curve for R Ara and found significant evidence of a steady period change due to mass transfer. The ephemeris curve is shown in Fig. 1.

The IUE data taken in 1989 provide over 4.5 days of consecutive images which are ideal for creating a light curve and analyzing phase-dependent variations. The two prominent outside-of-eclipse dips must be due to something cool eclipsing the primary star because they both become shallower at longer wavelengths, as shown in Fig. 2. Also shown in Fig. 2 are the models that best fit the light curves. These models approximate the effects of an eccentric accretion structure eclipsing the primary star by placing large cool clouds in the line of sight. The model is restricted to two circular clouds, but does a fair job of fitting the data at both wavelength regions. The two cool clouds represent the locations where the eccentric accretion structure is closest to the primary star. Due to the system's orbital inclination (and any additional inclination of the accretion structure itself relative to the orbital plane), we are presumably able to peer over the accretion structure where it is farther from the primary and are therefore able to see the star more directly. This appears to happen around phase 0.4. Fig. 3 displays an overhead view of the modeled components of the system, and illustrates the eccentric accretion structure.

\section{DISCUSSION AND OTHER SYSTEMS OF INTEREST}

R Ara was a good system to study because it had been observed in the past century but had been neglected over the past couple decades. Earlier studies indicated photometric variations and hinted at mass exchange as the cause, which is why it was considered in the study reviewed

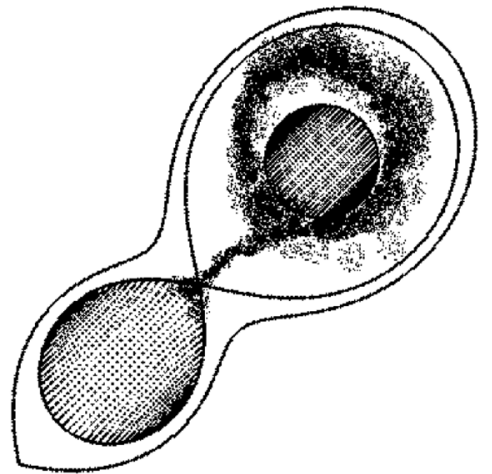

Fig. 3. An overhead view of the model of R Ara, illustrating the eccentric accretion structure. Reprinted from Reed et al. (2010).

in this paper. There are other such systems, studied in the past but neglected lately, that could also be candidates for observing similar effects of eccentric accretion $-\mathrm{Y}$ Psc $\left(\mathrm{P}_{\text {orb }}=\right.$ 3.7 days $)$ and RV Oph ( $\mathrm{P}_{\text {orb }}=3.9$ days $)$ to name a couple. The latest light curve study of RV Oph, by Walter (1970), clearly shows outside-of-eclipse dips similar to those seen in R Ara.

As discussed by Richards \& Ratliff (1998), short-period Algols tend to undergo direct mass transfer while longerperiod systems tend to build up stable accretion disks. The intermediate-period Algol systems will oscillate between states of direct impact and indirect accretion. It is in these intermediate-period systems that we would expect to find unstable, variable, and eccentric accretion structures.

A problem with building light cures for these systems from the ground is that it takes several weeks to months or even years to complete them. This complicates the analysis of phase-dependent variations with secular variations due to the variable nature of the accretion structure. The IUE data for R Ara were taken from space and eliminated this problem by providing continuous observations over one entire orbital cycle.

New space-based missions, such as the Kepler Observatory, are easily able to continuously observe binary systems over not just one orbit, but over multiple consecutive orbits. These advances in observational techniques could provide enough information to study the dynamics of variable accretion structures. We would expect an eccentric accretion structure to precess over time, which could actually be one factor contributing to secular variations. Kepler-quality data could enable us to model the shape of the accretion structure and also watch it change over time. Figs. 4 and 5 show the Kepler light curves (courtesy of Villanova University) for two system that are possible candidates for studying variable, transient, or eccentric accretion. They 


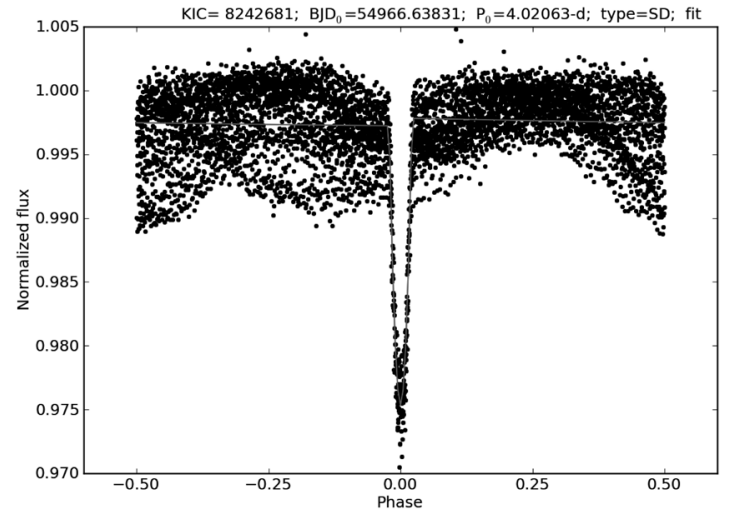

Fig. 4. A Kepler light curve of a binary star with an orbital period of 4.0 days. Reprinted from the Kepler eclipsing binary catalog (http://keplerebs. villanova.edu).

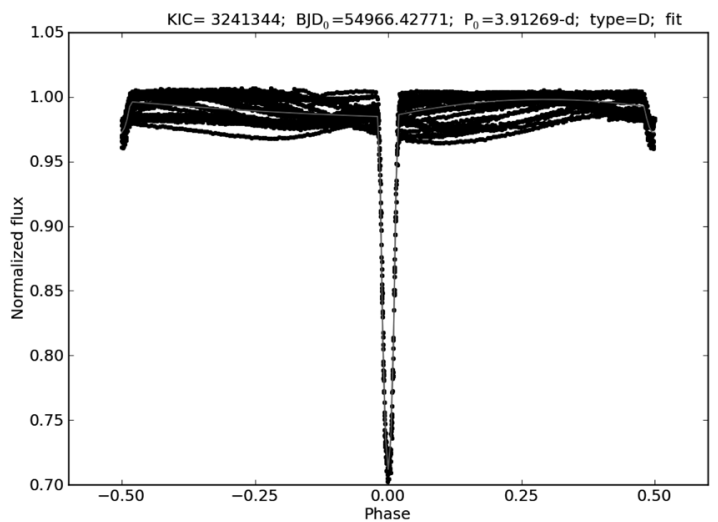

Fig. 5. A Kepler light curve of a binary star with an orbital period of 3.9 days. Reprinted from the Kepler eclipsing binary catalog (http://keplerebs. villanova.edu).

are KIC $3241344\left(\mathrm{P}_{\text {orb }}=3.9\right.$ days $)$ and KIC $8242681\left(\mathrm{P}_{\text {orb }}=\right.$ 4.0 days). It can be seen that there are outside-of-eclipse variations that may be due to accretion material eclipsing the star(s). Whatever the case, there are secular variations in both systems that occur from one orbit to the next and could provide information about short-term variability whether it is due to the precession of an eccentric accretion disk or not.

Finally, it should be noted that better models might be possible if we could incorporate an entire accretion structure, in addition to the primary and secondary stars, into a synthetic light curve generator. Most, if not all, of the available light curve modeling programs allow only for either two stars, or one star and an accretion disk (such as is the case with cataclysmic variables).

\section{REFERENCES}

Banks T, Light curves for R Arae, IBVS, 3455 (1990).

Forbes M, Budding E, Priestly J, Photometric fluctuations in the light curves of R Arae, IBVS, 3278 (1988).

Nield KM, Priestly J, Budding E, UBV observations of R Arae, IBVS, 2941 (1986).

Nield KM, Observations of analysis of the southern binary R Arae, Ap\&SS, 180, 233-252 (1991). http://dx.doi. org/10.1007/BF00648180

Reed PA, A 116 year record of mass transfer in R Arae, IBVS, 5975 (2011).

Reed PA, McCluskey GE Jr, Kondo Y, Sahade J, Guinan EF, et al., Ultraviolet study of the interacting binary star R Arae using archival IUE data, MNRAS, 401, 913-923 (2010). http://dx.doi.org/10.1111/j.1365-2966.2009.15741.x

Richards MT, Ratliff MA, Hydrodynamic simulations of $\mathrm{H} \alpha$ emission in Algol-type binaries, ApJ, 493, 326-341 (1998). http://dx.doi.org/10.1086/305087

Sahade J, The spectrum of the eclipsing variable R Arae, ApJ, 116, 27-34 (1952). http://dx.doi.org/10.1086/145590

Walter K, The Algol system RV Ophiuchi and the variations of its light curve with time, A\&A, 5, 140-148 (1970). 